\title{
XI. Ueber den Ardennit und über eine Methode zur Scheidung der Vanadinsäure von Thonerde und Eisenoxyd; von Anton Bettendorff.
}

In diesen Annalen Bd. CXLIX veröffentlichte ich gemeinsam mit Hrn. Prof. Dr. von Lasaulx einige Analysen des Ardennits, eines Minerals, das durch seinen Isomorphismus mit dem Ilvait und einen hohen Vanadinsäuregehalt von Interesse ist. Wir haben damals die Schwierigkeit der Trennung von Vanadinsäure und Thonerde hervorgehoben, da die beiden Substanzen ein ähnliches Verhalten zu einander zeigen, wie die Phosphorsäure zur Thonerde.

Seit jener Zeit habe ich eine leicht auszufübrende und scharfe Trennungsmethode für beide Substanzen aufgefunden und ist das ein Grund gewesen zur Ausführung neuer Analysen des Ardennits. Eine weitere Veranlassung dazu gab die Analyse des Hrn. Pisani i ${ }^{1}$, die einen Arsensäuregehalt von 6,35 Proc. in dem Minerale nachweist.

Mit Material zur Untersuchung war ich durch die Güte des Hrn. von Lasaulx reichlich versehen. Es fiel bei demselben zunächst die Verschiedenheit der Färbung auf. Wăhrend zu den früheren Untersuchungen nur dunkelbraune Sthcke gedient hatten, gebot ich jetzt uber solche vom undurchsichtigen hellen Schwefelgelb bis zum dunklen durchsichtigen Kolophoniumbraun.

Durch Auswabl der hellsten und dunkelsten Stücke bildete ich zwei Proben, die zu den folgenden Analysen gedient haben. No. I war hell schwefelgelb und undurchsichtig in dünnen Splittern, No. 2 war kolophoniumbraun und durchsichtig.

Bei den Bestimmungen des spec. Gewichtes dieser Proben wurden folgende Werthe gefunden.

1) Compt. rend. T. 75, p. 1542 und T. 77, p. 329. 


\begin{tabular}{|c|c|c|c|c|c|}
\hline Pycnometer leer & . & $\begin{array}{l}\text { No. I. } \\
5,3674 \mathrm{G}\end{array}$ & & $\begin{array}{l}\text { No. II. } \\
5,3668\end{array}$ & \\
\hline Pycnometer + Mineral . & - & 10,0168 & $\pi$ & 9,8844 & \\
\hline Pycnometer + Mincral - & - Wasser & 11,5055 & $\%$ & 11,7046 & $\mathrm{~T} .=17^{0}, 0 \mathrm{C}$. \\
\hline Pycnometer + Wasser & & 8,4275 & $n$ & 8,4275 & \\
\hline
\end{tabular}

Aus diesen Zahlen berechnet sich das spec. Gewicht für No. I zu 3,656, für No. II zu 3,643 .

Die Zerlegung des Ardennits wurde wie bei den früheren Analysen mit Soda bewirkt, der Gang der Analyse war folgender.

Nach Abscheidung der Kieselsäure wurde durch Schwefelwasserstoff das Arsen gefällt, in arsensaure AmmonMagnesia übergeführt und als solche gewogen. Das arsenfreie Filtrat wurde mit Ammon und Schwefelammon versetzt und dadurch die Schwefelverbindungen des Eisens, Vanadins, Mangans und die Thonerde entfernt. Das Filtrat enthielt kein Sulfosalz des Vanadins, der darin befindliche Kalk und die Magnesia wurden in üblicher Weise ermittelt. Der Schwefelammoniumniederschlag wurde in wenig Chlorwasserstoffsäure und einigen Tropfen Salpetersäure gelöst, zur Trockne gedampft, dann zur vollständigen Ueberführung des Vanadins in Vanadinsäure mit Salpetersäure befeuchtet und abermals trocken gedampft. Mit wenigen Tropfen Salpetersäure angesäuertes Wasser löst den durcb Vanadsäure braun gefürbten Abdampfungs-Rückstand zu einer klaren. mehr oder minder gelbgefärbten, Flüssigkeit auf. Aus derselben wurde mit kohlensaurem Baryt, Thonerde, Eisenoxyd und Vanadsäure abgeschieden ${ }^{1}$ ), Manganoxydul blieb in Lösung und wurde in üblicher Weise als Manganoxyd-Oxydul bestimmt.

Die Trenuung der Vanadsäure von der Thonerde beruht nun auf folgender Thatsache.

1) In der ersten Abhandlung über den Ardennit habe ich angegeben, dafs auch das Tetroxyd des Vanadins durch kohlensauren Baryt gefällt werdc. Es ist das nicht der Fall, wie mir neue Versuche gezeigt haben. 
Wird eine Lösung der beiden Stoffe mit Ammon versetzt, so fällt gelbe vanadsaure Thonerde, ein Ueberschul's von Ammon entzieht ihr die Vanadsäure nicht. Fügt man zu der gelben Fällung phosphorsaures Ammon, so wird nach Erwärmung auf dem Wasserbade der gelbe Niederschlag rein weifs und die Flüssigkeit enthält vanadsaures Ammon. Die vanadsaure Thonerde hat sich mit dem phosphorsauren Ammon vollständig zu phosphorsaurer Thonerde und löslichem vanadsauren Ammon umgesetzt. Auch bei Gegenwart von Eisenoxyd findet diese Umsetzung statt. Die phosphorsaure Thonerde bildet einen schleimigen Niederschlag, der sich rasch absetzt aber nicht auf dem Filtrum auswaschen läfst. Man mul's ihn durch Decantiren reinigen und das Absetzen durch jeweiligen Absatz einiger Tropfen Chlorammonlösung befördern.

Der Fortgang der Analyse war nun folgender:

Der Niederschlag von Thonerde, Eisenoxyd und vanadsaurem Baryt wird in wenig Chlorwasserstoff säure gelöst, der Baryt durch Schwefelsäure entfernt und dann einige Tropfen Phosphorsäure ${ }^{1}$ ) zugefügt. Man erhitzt auf dem Wasserbade und fält phosphorsaure Thonerde und phosphorsaures Eisenoxyd mit Ammon. Alle Vanadsäure bleibt als Ammon in Lösung. Ist der Niederschlag noch gelb gefärbt, so war zu wesig Phosphorsäure vorhanden, man löst daher abermals und verfährt nach Zusatz von Phosphorsăure wie vorhin. Durch Absetzenlassen und Abgiefsen durch ein Filtrum wird das vanadsaure Ammon von dem Niederschlag getrennt und der letztere durch Decantiren ausgewaschen. Das gesammelte Waschwasser wird eingedampft, wobei meist noch eine sehr kleine Menge phosphorsaure Thonerde sich abscheidet, die sorgfältig entfernt und der obigen Fällung beigefügt wird. Die auf ein kleines Volumen eingedampfte Flüssigkeit ist hellgelb gefärbt, wie eine schwache Lösung von chromsaurem Kali.

1) Durch Findampfen von wasserfreier Phosphorsäure mit Salpetersäure, und nachheriges Einleiten von Schwefelwasserstoffgas zur Entfernung der Arsensäıre, erhalten. 
Sie enthält das vanadsaure Ammon in der gelben Modification, welches sich bei genügender Concentration als hellgelbes Krystallpulver absetzt. Man fällt nun die Vanadsäure nach Angabe von Berzelius durch Sättigen der gelben Flüssigkeit mit Chlorammon, wodurch nach einigen Tagen der grölste Theil als vanadsaures Ammon abgeschieden wird. Es ist aber nach meinen Erfahrungen nicht möglich alle Vanadsäure auf diese Weise zu fällen. Ein Theil bleibt selhst bei Gegenwart von Alkobol, den von Hauer empfohlen hat, in Lösung. Eine vollständigere Abscheidung erfolgt, wenn zu der phosphorsäurehaltigen Lösung der Vanadsäure Schwefelammon gefügt und dann mit Essigsäure schwach eingesäuert wird. Anfangs entsteht keine Fällung, bei schwachem Erwärmen und Stehenlassen erfolgt in einigen Stunden Abscheidung von braunem Schwefelvanadin. Die Flüssigkeit enthält die Phosphorsäure und nur Spuren von Vanadsäure. Durch Glüben wird das Schwefelvanadin in Vanadsäure übergeführt. Sic war bei den Analysen beider Ardennit-Proben ohne jeden Rückstand in Ammon löslich.

Der Niederschlag von phosphorsaurer Thonerde und Eisenoxyd wurde in Salpetersäure gelöst, mit molybdänsaurem Ammon die Phosphorsäure entfernt und dann mit Ammon, Thonerde und Eisenoxyd gefällt. Um dieselben gänzlich frei von Molybdänsäure zu erhalten, wurde die Fällung nochmals in Chlorwasserstoffsäure gelöst und mit Ammon und Schwefelammon Thonerde und Eisenoxyd abgeschieden, die nun in der gewöhnlichen Weise getrennt und bestimmt wurden.

Es ergaben sich auf diese Weise für die Bestandtheile der beiden Mineralproben folgende Werthe.

Probe No. I. Angewandte Menge 1,0142 Grm. bei $105^{\circ} \mathrm{C}$. getrocknet. Gefunden: Kieselsäure 0,2790. Arsensaure Ammon-Magnesia bei $100^{n} \mathrm{C}$. getrocknet 0,1566 . Kupferoxyd 0,0018. Kalk 0,0186. Phosphorsaure AmmonMagnesia 0,0361. Manganoxyd-Oxydul 0,3339. Vanadin-

Poggendorff's Annal. Bd. CLX. 
săure 0,0054. Thonerde 0,2299. Eisenoxyd 0,0117. Glühverlust durch Erhitzen auf der Gebläselampe: 0,9099 Grm. Ardennit gaben 0,0467 Verlust.

Probe No. II. Angewandte Menge 1,0302 Grm. bei $105^{\circ} \mathrm{C}$. getrocknet. Gefunden: Kieselsäure 0,2869. Arsensaure Ammon-Magnesia bei $100^{\circ} \mathrm{C}$. getrocknet 0,0470 . Kalk 0,0224. Phosphorsaure Ammon-Magnesia 0,0862. Manganoxyd-Oxydul 0,2958. Vanadinsäure 0,0948. Thonerde und Eisenoxyd 0,2495. Glühverlust durch Erhitzen auf der Gebläselampe: $\mathbf{0 , 6 4 8 0} \mathrm{Grm}$. Ardennit gaben 0,0331 Verlust.

Aus den vorstehenden Angaben berechnen sich folgende procentische Werthe:

\begin{tabular}{|c|c|c|}
\hline Kieselsäure & $\begin{array}{l}\text { Probe No. I. } \\
. \quad 27,50\end{array}$ & $\begin{array}{c}\text { Probe No. I } \\
27,84\end{array}$ \\
\hline Thonerde & - 22,76 & \multirow{2}{*}{24,22} \\
\hline Eisenoxyd & 1,15 & \\
\hline Manganoxydu & . 30,61 & 26,70 \\
\hline Kalk & 1,83 & 2,17 \\
\hline Magnesia . & 1,38 & 3,01 \\
\hline Kupfer & 0,17 & 0,00 \\
\hline Vanadinsäure & 0,53 & 9,20 \\
\hline Arsensäure & 9,33 & 2,76 \\
\hline Wasser . & . $\quad 5,13$ & 5,01 \\
\hline & 100,39 & 0,91 . \\
\hline
\end{tabular}

Der Kieselsãuregehalt der beiden Analysen weicht erheblich von dem früher gefundenen, 29,60, 29,89 ab. Es ist das nicht auffallend, da der Ardennit meist von feinen Quarzgängen durchzogen ist. Bei der reichen Menge an Material, welches mir zu Gebote stand, war es möglich diesen Quarz besser zu entfernen als bei den ersten Analysen, wo nur über eine geringe Menge verfügt wurde.

Es zeigt sich nun, dafs in der That der Ardennit Arsensäure enthält und zwar in wechselnder Menge, dafs 
wie es scheint Vanadinsäure und Arsensäure sich im Ardennit gegenseitig vertreten, wodurch die Verschiedenheit der Färbung bedingt wird. Es hat mich dieses veranla/st, noch einige Arsenbestimmungen auszuführen und habe ich je nach der Farbe auch wechselnden Gehalt an Arsensäure gefunden und $z$ war einen um so geringeren, je dunkler das Mineral von Farbe war. Es wurden folgende procentische Mengen erhalten: 1,83, 2,31; 2,53, 2,98, 6,64. Die ersten von mir und Hrn. Prof. Lasaulx ausgeführten Analysen waren mit einem sehr dunklen Material vorgenommen worden, wie es für diese Analysen nicht mehr vorlag, ich halte defshalb an der Meinung fest, dafs dieses Material frei von Arsensäure war. Es steht dem kein Grund entgegen, da sich Vanadin- und Arsensäure gegenseitig vertreten und wir, wie Analyse I zeigt, sogar einen Ardennit haben, der Vanadinsäure in sehr untergeordneter Menge enthält. Man könnte defshalb einen Vanad- und einen Arsen-Ardennit unterscheiden.

\section{Ueber die Zusammensetzung des Speis- kobalts und verwandter. Mineralien; von C. Rammelsberg.}

Vor einiger Zeit hat Groth ') mitgetheilt, dals an den Krystallen von Speiskobalt Flächen vorkommen, welche die pyritoëdrische Hemiedrie des Minerals aulser $Z_{\text {weifel }}$ setzen. Denn er beobachtete das Pentagondodekaëder $a: 3 a: \infty a$ und ein gebrochenes Pentagondodekaëder, vielleicht $a: \frac{1}{3} a: \frac{1}{8} a$, neben anderen nicht genau bestimmbaren Pentagondodekaëdern. Aufserdem fand er, dafs die Krystalle des Speiskobalts sich gegen Kupfer thermo-elek1) Pogg. Ann. Bd. 152, S. 243. 